\title{
$\underline{\mathbf{P}-127}$
}

\section{Triterpenes from the Stems of Syzygium Filiforme var. Filiforme}

\author{
Mohd Hafiz Ahmad ${ }^{1,2, *}$, Nor Hadiani Ismail ${ }^{1,2}$, Humera Naz ${ }^{1,3}$ \\ ${ }^{1}$ Atta-ur-Rahman Institute for Natural Products Discovery (RiND), Universiti Teknologi MARA (UiTM); ${ }^{2}$ Faculty of \\ Applied Sciences, Universiti Teknologi MARA, 40450 Shah Alam, Selangor; ${ }^{3}$ Faculty of Pharmacy, Universiti Teknologi \\ MARA, Puncak Alam Campus, 42300 Puncak Alam, Selangor; E-mail: hafiz99414@yahoo.com
}

Syzigium filiforme var. filiforme belongs to the family Myrtaceae. This family comprises of 121 genera and $3800-5800$ species of shrubs or trees. They are widely spread in tropical and subtropical regions of the world. This family is a rich source of essential oils and few sesquiterpenes, triterpenoides, ellagic acids and flavanols are also reported from it. The essential oils of most species exhibited antifungal and antibacterial properties. It seems that the bioactivity is associated with the presence of phenolic compounds or with a high level of oxygenated terpenes. Betulinic acid, a triterpenoid isolated from the methanolic extract of the leaves of Syzigium claviflorum, has potent inhibitory activity against human immunodeficiency virus type 1 (HIV-1). The oils of some species also demonstrated cytotoxic, antioxidant, antilarvae, anti-inflammatory and analgesic activities. The objective of the present study is to isolate bioactive compounds from the stems of Syzygium filiforme var. filiforme. Dried stems of the plant were ground into fine powder using a cutter mill. The powder was successively extracted with hexane, dichloromethane and methanol. The dichloromethane extract was fractionated by using vacuum liquid chromatography and two triterpenes were successfully isolated from fractions no. 7 and no. 11 by using radial chromatography technique. The compounds were proposed as alphitolic acid (7) and arjulonic acid (11) by using spectroscopic techniques including NMR and LC-MS and comparison with literature. 